\title{
Clinical predictors of hypoxaemia in Gambian children with acute lower respiratory tract infection: prospective cohort study
}

\author{
Stanley Usen, Martin Weber, Kim Mulholland, Shabbar Jaffar, Anslem Oparaugo, Charles Omosigho,
} Richard Adegbola, Brian Greenwood

\section{Medical Research \\ Council \\ Laboratories, \\ PO Box 273, Fajara, \\ Gambia \\ Stanley Usen, \\ research clinician \\ Shabbar Jaffar, \\ unit statistician \\ Charles Omosigho, \\ research clinician \\ Anslem Oparaugo, \\ research clinician \\ Richard Adegbola, microbiologist \\ Global Programme on Vaccine and \\ Immunisation, \\ World Health \\ Organisation, 1211 \\ Geneva 27, \\ Switzerland \\ Martin Weber, \\ research clinician \\ Kim Mulholland, \\ paediatrician \\ London School of \\ Hygiene and \\ Tropical Medicine, \\ London \\ WC1E 7HT \\ Brian Greenwood, \\ director \\ Correspondence to: Dr Usen \\ susen@mrc.enda.sn}

BMJ 1999;318:86-91

\begin{abstract}
Objectives To determine clinical correlates and outcome of hypoxaemia in children admitted to hospital with an acute lower respiratory tract infection. Design Prospective cohort study. Setting Paediatric wards of the Royal Victoria Hospital and the hospital of the Medical Research Council's hospital in Banjul, the Gambia.

Subjects 1072 of 42848 children, aged 2 to 33 months, who were enrolled in a randomised trial of a Haemophilus influenzae type b vaccine in the western region of the Gambia, and who were admitted with an acute lower respiratory tract infection to two of three hospitals.
\end{abstract}

Main outcome measures Prevalence of hypoxaemia, defined as an arterial oxygen saturation $<90 \%$ recorded by pulse oximetry, and the relation between hypoxaemia and aetiological agents.

Results 1072 children aged 2-33 months were enrolled. Sixty three $(5.9 \%)$ had an arterial oxygen saturation $<90 \%$. A logistic regression model showed that cyanosis, a rapid respiratory rate, grunting, head nodding, an absence of a history of fever, and no spontaneous movement during examination were the best independent predictors of hypoxaemia. The presence of an inability to cry, head nodding, or a respiratory rate $\geqslant 90$ breaths/min formed the best predictors of hypoxaemia (sensitivity $70 \%$, specificity $79 \%$ ). Hypoxaemic children were five times more likely to die than non-hypoxaemic children. The presence of malaria parasitaemia had no effect on the prevalence of hypoxaemia or on its association with respiratory rate.

Conclusion In children with an acute lower respiratory tract infection, simple physical signs that require minimal expertise to recognise can be used to determine oxygen therapy and to aid in screening for referral. The association between hypoxaemia and death highlights the need for early recognition of the condition and the potential benefit of treatment.

\section{Introduction}

Acute lower respiratory tract infections are a major cause of morbidity and mortality among children in developing countries, accounting for about 30\% of mortality in children under 5 years of age. ${ }^{12}$ Most of these deaths occur at home before children enter the healthcare system. ${ }^{3}$

Use of simple algorithms for the case management of children with acute lower respiratory tract infections has resulted in a significant reduction in mortality from pneumonia. ${ }^{4}$ Oxygen therapy improves the outcome of children with moderate or severe acute lower respiratory tract infection and, in those with hypoxaemia, the severity of hypoxia correlates with outcome. ${ }^{56}$ However, in countries with limited resources-for example, the Gambia-oxygen is not always freely available. Thus it is important to have rational guidelines both for the use of oxygen and for the referral of patients to specialist hospitals.

Pulse oximetry is a non-invasive and accurate method of measuring arterial oxygen saturation. ${ }^{7}$ It is a useful predictor of hypoxaemia and pneumonia. ${ }^{89}$ Pulse oximeters are, however, not available in most health centres in developing countries. For this reason clinical signs that best predict pneumonia, hypoxaemia, or mortality have been evaluated in earlier studies. ${ }^{6}{ }^{10-13}$ No single sign has been found to be a reliable predictor of hypoxaemia. Cyanosis is the most specific predictor and the best clinical correlate of arterial oxygen saturation, but it is difficult to detect. ${ }^{6}{ }^{83-19}$ In some studies, mainly those undertaken at high altitude, ${ }^{68917}$ a rapid respiratory rate was found useful as a predictor of hypoxaemia, but this was not the case in all studies. ${ }^{1520}$ Moreover, children with malaria and anaemia, who are not necessarily hypoxaemic, may present with a rapid respiratory rate that is attributable to fever or cardiac failure. In order to clarify further the predictive value of clinical signs in hypoxaemia, a Kenyan study used a logistic regression model. This study found that the presence of chest retraction, a respiratory rate $\geqslant 70$ breaths $/ \mathrm{min}$, or grunting were the best predictors of hypoxaemia. ${ }^{6}$ Chest retractions have been found useful as a predictor of hypoxaemia in children with bronchiolitis. ${ }^{15}{ }^{16}$ In an earlier study from the Gambia, we found that the combination of cyanosis, head nodding (visible movement of the head with each inspiration), and not crying during examination identified over a half of hypoxaemic children. ${ }^{21}$ The study was, however, small and the investigators were not blinded to one of the two control groups. We analysed data collected during a large trial of a vaccine for the prevention of infant pneumonia, to study further which clinical signs predict hypoxaemia.

\section{Subjects and methods}

\section{Selection criteria}

Our study was undertaken in the context of an efficacy trial of a Haemophilus influenzae type b (Hib) conjugate vaccine in the western region of the Gambia from March 1993 to December $1995 .^{22}$ During the course of the trial, 42848 infants (about $90 \%$ of children resident in the study area) were enrolled when they presented to a vaccination clinic for routine immunisation at the age of 2 months. If any of the children in the trial cohort presented to two of three study hospitals, they were evaluated by one of our study physicians for features compatible with an invasive bacterial disease. Children in whom this was considered a possible diag- 
nosis were managed appropriately. Children in the trial cohort who were eligible for inclusion in our study were those admitted with pneumonia or any other form of acute lower respiratory tract infection to either the MRC's hospital at Banjul-a primary and secondary healthcare institution serving a periurban population-or to the Royal Victoria Hospital-the main referral hospital in the Gambia's capital, Banjul. We excluded children aged $<2$ months (they were too young to be enrolled in the vaccine trial), those with signs of structural heart disease, those with Down's syndrome, and those who had been included in a previous case-control study of hypoxaemia. As almost all children resident in the study area were enrolled in the vaccine trial, and as no other selection criteria were imposed, it is likely that the children included in our study were representative of children in the local community with acute lower respiratory tract infections. As it is unlikely that Hib vaccination would influence the association between clinical signs and hypoxaemia, this variable was not included in analyses.

\section{Protocol}

Our physicians recorded signs and symptoms using standardised forms. Training sessions were held before and throughout the study period to ensure uniformity of record keeping between observers. Mothers were asked first about their children's symptoms and then questioned on the presence of specific symptoms such as cough, fever, refusal to feed or drink, rapid or difficult breathing, abnormal sleepiness, arousability, or irritability. The physician then examined the child for cyanosis, impaired consciousness, inability to eat or drink, chest retraction, head nodding, grunting, and the presence of wheeze, rhonchi, crepitations, or bronchial breath sounds. The physician documented the child's respiratory rate, pulse rate, weight, and height. On admission the child's arterial oxygen saturation was measured with an appropriately sized sensor and a Nellcor N200 pulse oximeter (Hayward, CA, USA) placed on a toe or finger, while the patient breathed room air. The measurement selected was that which had been stable for at least three minutes. Further measurements of arterial oxygen saturation were undertaken as part of the management of children whose clinical condition deteriorated or who received oxygen therapy. Blood samples were taken from all children in the trial cohort. These samples were cultured. Lung aspiration was performed when chest $x$ rays showed consolidation adjacent to the chest wall, and pleural aspirations were done in cases of pleural effusion. Nasopharyngeal aspirates were obtained from children admitted on a working day, and these were examined for respiratory syncytial virus by immunofluorescence. ${ }^{23}$ Chest $x$ rays, obtained whenever possible, were assessed at the end of the study by one of the physicians (SU, MW, AO, CO, KM) who did not know the clinical details of the case. The classification of parenchymal changes has been described previously. ${ }^{21}$

Hypoxaemia was defined as an arterial oxygen saturation $<90 \%$ recorded by pulse oximetry. A cut off point of $90 \%$ was chosen because saturations below this are regarded by most clinicians as indicating clinically significant hypoxaemia. An arterial oxygen saturation of $90 \%$ generally corresponds to an arterial oxygen tension of 60-70 $\mathrm{mm} \mathrm{Hg}$, although this relation is affected by factors such as temperature, $\mathrm{pH}$, altitude, and age. Below an arterial oxygen tension of $60-70 \mathrm{~mm} \mathrm{Hg}$ the haemoglobin oxygen dissociation curve falls steeply such that further decreases in arterial oxygen tension are associated with greater decreases in arterial oxygen saturation. ${ }^{24}$

Consent was obtained from the parents at enrolment of their child into the vaccine trial. No additional consent was sought for this study as the protocol was within standard management guidelines already in use in the Gambia. The study was approved by the Gambian Government and the MRC Laboratories' ethical committee.

\section{Statistical analysis}

We compared discrete clinical signs between hypoxaemic and non-hypoxaemic children by using the $\chi^{2}$ test with Yates correction, or by using Fisher's exact test if the frequencies were small. We compared continuous variables by using Student's $t$ test or a Wilcoxon rank sum test, as appropriate. Signs and symptoms that showed a significant association with hypoxaemia in the univariate analyses at a significance level of $\leqslant 0.05$, together with those that had been reported as important predictors in previous literature, were included in multivariate logistic regression analyses that assessed their independent utility in predicting hypoxaemia. We repeated modelling using variables in the categories of complaints, general status, respiratory signs, and other signs. For each category we built up models consecutively, starting with the most significant variable, until no further variables reached significance. We then removed variables from the multivariate model one at a time to see if they remained significant in the presence of all other variables. We then investigated together significant and independent predictors of hypoxaemia identified in each category, from which we acquired a final set of predictors. We compared different models with one another by change in deviance. We then calculated the diagnostic values of various combinations of the variables identified in the logistic regression modelling, and others that had been reported in the literature. For the analysis we used EPI Info version 6 and SAS for Windows.

\section{Results}

\section{Characteristics of the study population}

Two thousand and ninety seven (4.9\%) of the 42848 children were evaluated by our study physicians, 1114 $(53.1 \%)$ of whom were considered to have an acute lower respiratory tract infection that required hospital admission. We excluded $23(2.1 \%)$ children who had either congenital heart disease or Down's syndrome, and $19(1.7 \%)$ children who had been enrolled in a previous case-control study. The remaining 1072 (96.2\%) children form the basis of this analysis. Sixty three $(5.9 \%)$ children had hypoxaemia.

Males comprised 55.0\% (555/1009) of the children in the non-hypoxaemic group and $46.0 \%(29 / 63)$ in the hypoxaemic group $(\mathrm{P}=0.2)$. The median age was 8 months for non-hypoxaemic children and 9 months for hypoxaemic children. The median oxygen saturation was $86 \%$ (interquartile range $80-88 \%$ ) for hypoxaemic children and 97\% (95-99\%) for non- 
hypoxaemic children was. The duration of hospital stay was significantly longer for hypoxaemic children (median 5 days; interquartile range 2-8 days) than for non-hypoxaemic children (median 4 days; 2-6 days) $(\mathrm{P}=0.03)$. Thirty six of the $1072(3.4 \%)$ children died; $28(2.6 \%)$ in the non-hypoxaemic group and $8(12.7 \%)$ in the hypoxaemic group (relative risk 4.6, 95\% confidence interval 2.2 to 9.6$)(\mathrm{P}=0.0007)$.

Chest radiography was performed on $712(66.4 \%)$ children; chest $x$ rays were obtained from $36(57.1 \%)$ of the hypoxaemic children and from $676(67 \%)$ of the non-hypoxaemic children. Lobar consolidation was present in $12(33.3 \%)$ hypoxaemic children and in 158 (23.4\%) non-hypoxaemic children $(\mathrm{P}=0.6)$. Pneumonia, hyperinflation, and pleural effusions were found in similar proportions in the chest $x$ rays from both hypoxaemic and non-hypoxaemic children.

\section{Aetiological agents and hypoxaemia}

One hundred and seventy seven invasive bacterial isolates were identified from the 1072 children. Streptococcus pneumoniae was the most commonly isolated pathogen and was found in $8.5 \%(\mathrm{n}=91)$ of the children. $H$ influenzae type b was isolated from $2.2 \%$ $(\mathrm{n}=24)$ of the children, Staphylococcus aureus from $2.1 \%$ $(\mathrm{n}=23)$, Salmonella spp from $1.2 \%(\mathrm{n}=13)$, and $H$ influenzae non-type b from $0.6 \%(\mathrm{n}=6)$. Pneumococci were isolated from 10 of the $63(15.8 \%)$ hypoxaemic children and from 81 of the 1009 (8.1\%) nonhypoxaemic children $(\mathrm{P}=0.05)$. The isolation rates of other bacteria did not differ significantly between hypoxaemic and non-hypoxaemic children.

Of the 996 children who underwent nasopharyngeal aspiration, $101(10.1 \%)$ were positive for respiratory syncytial virus by immunofluorescence. This infection was not associated significantly with hypoxaemia (6/60 (10.0\%) versus 95/936 (10.1\%) $\mathrm{P}=0.9$ ). None of the five children from whom both respiratory syncytial virus and bacteria were isolated were hypoxaemic.

\section{Malaria and hypoxaemia}

Blood films were examined for malaria parasites in $1040(97.0 \%)$ children. Malaria parasitaemia was found in similar proportions of children who were hypoxaemic (4/60, 6.7\%) and non-hypoxaemic (103/980, $10.5 \%)(\mathrm{P}=0.5)$. Neither the presence of pallor nor a temperature $\geqslant 38^{\circ} \mathrm{C}$ was associated significantly with hypoxaemia (14/63 (22.2\%) versus 161/1009 (15.0\%); relative risk $1.39,95 \%$ confidence interval 0.86 to 2.26 , $\mathrm{P}=0.13$ and $49 / 63$ (77.8\%) versus $817 / 1009$ (81.0); $0.96,0.84$ to $1.10, \mathrm{P}=0.6$ respectively).

\section{Symptoms and signs associated with hypoxaemia}

\section{Univariate analysis}

The box shows the symptoms and signs evaluated in the children. Of the complaints volunteered by mothers, only the absence of a history of fever had a significant association with hypoxaemia; a history of fever was volunteered by 946 of the 1009 (93.7\%) mothers of non-hypoxaemic children and 52 of 63 $(83 \%)$ mothers of hypoxaemic children $(\mathrm{P}=0.001)$. A history of cough, fast breathing, difficulty in breathing, diarrhoea, vomiting, abdominal pain, chest pain, or convulsions was reported spontaneously with a similar

\author{
Variables analysed as predictors of hypoxaemia \\ in children admitted with an acute lower \\ respiratory tract infection \\ Complaints \\ Cough \\ Breathing difficulty \\ Chest pain \\ Fast breathing \\ Fever \\ Excessive crying \\ Diarrhoea \\ Soft tissue swelling \\ Convulsion \\ Severely reduced feeding \\ Inability to drink or feed \\ Irritability \\ Unusually sleepy \\ Examination \\ General status: \\ Appearance: very sick, mildly sick, well \\ Movement during examination \\ Abnormally sleepy \\ Quality or ability to cry while examined \\ Restless or irritable \\ Stridor \\ Respiratory signs: \\ Nasal flaring \\ Central cyanosis \\ Intercostal indrawing \\ Lower chest indrawing \\ Head nodding \\ Continuous grunting \\ Rapid respiratory rate \\ Crepitations \\ Wheeze \\ Rhonchi \\ Diminished breath sounds \\ Bronchial breath sounds \\ Other signs: \\ Pallor of palms or conjunctiva \\ Hepatomegaly $(>2 \mathrm{~cm})$ \\ Abnormal chest $x$ rays \\ Temperature $>38^{\circ} \mathrm{C}$ \\ Malaria parasitaemia \\ Nutritional status: weight for height, weight for age
}

frequency for both hypoxaemic and non-hypoxaemic children. No mother volunteered a history of bluish discoloration of their child's tongue or nail beds.

Table 1 shows the distribution of prompted symptoms and clinical signs that were significantly associated with hypoxaemia. A prompted history from the mother of difficulty in breathing, fast breathing, or irritability was not associated with hypoxaemia. Respiratory signs such as cough heard during examination, severe intercostal indrawing, rhonchi, decreased air entry, or bronchial breath sounds were not significantly associated with hypoxaemia. In contrast, signs that evaluated the general status of the child, such as inability to feed or cry, were associated strongly with hypoxaemia.

\section{Sensitivity and specificity of clinical signs}

Table 1 shows the sensitivity and specificity of signs and symptoms in hypoxaemic and non-hypoxaemic children that were significantly associated with hypoxaemia. Head nodding was found to be a useful sign (sensitivity $57 \%$, specificity $85 \%$ ). Cyanosis, an inability to feed or cry, abnormal sleepiness, and no 
Table 1 Frequency of symptoms and signs in hypoxaemic and non-hypoxaemic children that were associated significantly with hypoxaemia. Values are numbers (percentages) unless stated otherwise

\begin{tabular}{|c|c|c|c|c|c|c|c|}
\hline Clinical features & $\begin{array}{c}\text { Hypoxaemic } \\
\text { children }(n=63)\end{array}$ & $\begin{array}{l}\text { Non-hypoxaemic } \\
\text { children }(n=1009)\end{array}$ & $P$ value & Sensitivity & Specificity & $\begin{array}{c}\text { Positive } \\
\text { predictive } \\
\text { value }\end{array}$ & $\begin{array}{c}\text { Negative } \\
\text { predictive value }\end{array}$ \\
\hline \multicolumn{8}{|l|}{ Complaints } \\
\hline Severely reduced feeding & $11(18)$ & $34(3)$ & $<0.001$ & 17 & 97 & 24 & 95 \\
\hline Abnormal sleepiness & $22(35)$ & $132(13)$ & $<0.001$ & 35 & 88 & 15 & 96 \\
\hline Absence of history of fever & $52(83)$ & $946(94)$ & 0.001 & 83 & 6 & 5 & 85 \\
\hline \multicolumn{8}{|l|}{ General status } \\
\hline Sickly appearance & $44(70)$ & $377(37)$ & $<0.001$ & 70 & 63 & 11 & 97 \\
\hline No spontaneous movement & $29(46)$ & $158(16)$ & $<0.001$ & 46 & 84 & 16 & 96 \\
\hline Inability to feed & $21(33)$ & $93(9)$ & $<0.001$ & 33 & 91 & 18 & 96 \\
\hline Impaired rousability & $31(49)$ & $237(23)$ & $<0.001$ & 49 & 77 & 12 & 97 \\
\hline Inability to cry & $8(13)$ & $46(5)$ & 0.01 & 13 & 95 & 15 & 95 \\
\hline \multicolumn{8}{|l|}{ Respiratory signs } \\
\hline Nasal flaring & $62(98)$ & $837(83)$ & 0.002 & 98 & 17 & 7 & 99 \\
\hline Cyanosis & $16(25)$ & $51(5)$ & $<0.001$ & 25 & 95 & 24 & 95 \\
\hline Head nodding & $36(57)$ & $158(16)$ & $<0.001$ & 57 & 85 & 19 & 97 \\
\hline Grunting continuously & $29(46)$ & $143(14)$ & $<0.001$ & 46 & 86 & 17 & 96 \\
\hline \multicolumn{8}{|l|}{ Respiratory rate (breaths/min): } \\
\hline$\geqslant 60$ & $51(81)$ & $536(53)$ & $<0.001$ & 81 & 47 & 9 & 98 \\
\hline$\geqslant 70$ & $34(54)$ & $206(20)$ & $<0.001$ & 54 & 80 & 14 & 97 \\
\hline$\geqslant 90$ & $8(13)$ & $29(3)$ & $<0.001$ & 13 & 97 & 22 & 95 \\
\hline Crepitations & $54(86)$ & $704(70)$ & 0.01 & 86 & 30 & 7 & 97 \\
\hline Wheeze & $27(43)$ & $283(28)$ & 0.02 & 43 & 72 & 9 & 95 \\
\hline \multicolumn{8}{|l|}{ Other signs } \\
\hline Liver $>2 \mathrm{~cm}$ & $22(35)$ & $184(18)$ & 0.002 & 35 & 85 & 11 & 95 \\
\hline
\end{tabular}

spontaneous movement during examination were specific but relatively insensitive signs.

Regression model-In a regression model, no spontaneous movement during examination (odds ratio $2.8,95 \%$ confidence interval 1.5 to 5.0$)$, head nodding $(2.9,1.6$ to 5.3$)$, cyanosis $(4.6,2.3$ to 9.3$)$, a respiratory rate $\geqslant 70$ breaths/ $\mathrm{min}(2.4,1.4$ to 4.3$)$, grunting (3.0, 1.3 to 6.8$)$, and an absence of a history of fever (3.1, 1.4 to 6.7$)$ were independent and significant predictors of hypoxaemia.

Sensitivity and specificity of combinations of clinical signs-We tested various combinations of signs described in the literature for their ability to predict hypoxaemia in our data set. In addition, we tested combinations of the significant independent predictors established in the regression analysis of our data set. In our setting with limited resources, a model with a high specificity is desirable. The presence of either cyanosis or head nodding was the most specific predictor. This model would have missed 20 out of 63 (31.7\%) hypoxaemic children, and its absence correctly identified eight out of 10 children who were not hypoxaemic. The addition of other signs, as in our earlier study, improved the sensitivity but compromised the specificity of our model..$^{21}$ Table 2 shows the results of these models. Because of difficulty in assessing cyanosis, we combined signs other than cyanosis until we obtained the most specific model that also identified the majority of hypoxaemic children. The presence of either head nodding, an inability to cry, or a respiratory rate $\geqslant 90$ breaths/min formed the best predictor. This model would have missed 19 out of 63 (30.2\%) hypoxaemic children of whom three $(15.8 \%)$ were cyanosed, four $(21.0 \%)$ had grunting respiration, and five (26.3\%) had pneumonia detected by radiology. One of these children died. Using this model, 216 of the 1009 $(20.1 \%)$ children whose arterial oxygen saturation was $\geqslant 90 \%$ would have received oxygen, $17(7.9 \%)$ of whom were cyanosed, 50 (23.2\%) had grunting respiration, 31 (14.4\%) had a positive blood culture result, and 12 $(5.6 \%)$ died. Thus many of these children were severely ill and might have benefited from oxygen therapy, even though one of their arterial oxygen saturation measurements was $\geqslant 90 \%$.

\section{Discussion}

Pneumonia is a serious disease in children, and hypoxaemia is the best indicator of both severe and potentially fatal pneumonia. ${ }^{6}$ Prompt recognition of

Table 2 Precision of different combination models in prediction of hypoxaemia in children

\begin{tabular}{|c|c|c|c|c|c|}
\hline Combination & Study & Sensitivity & Specificity & $\begin{array}{c}\text { Positive } \\
\text { predictive value }\end{array}$ & $\begin{array}{c}\text { Negative } \\
\text { predictive value }\end{array}$ \\
\hline $\begin{array}{l}\text { Inability to feed or drink or cyanosis or respiratory rate } \geqslant 70 \\
\text { breaths/min or severe chest indrawing }\end{array}$ & $\mathrm{WHO}^{5}$ & 80.9 & 62.5 & 11.9 & 98.1 \\
\hline $\begin{array}{l}\text { Respiratory rate } \geqslant 70 \text { breaths/min or grunting respiration or severe } \\
\text { intercostal indrawing (age }<12 \text { months) }\end{array}$ & Onyango et al ${ }^{6}$ & 84.2 & 63.3 & 11.3 & 98.6 \\
\hline $\begin{array}{l}\text { Grunting or respiratory rate } \geqslant 90 \text { breaths/min or cyanosis or abnormally } \\
\text { sleepy }\end{array}$ & Dyke et $\mathrm{a}^{19}$ & 73 & 72 & 13.9 & 97.7 \\
\hline Cyanosis or head nodding or inability to cry & Weber et $\mathrm{al}^{21}$ & 71.4 & 77.1 & 16.3 & 97.7 \\
\hline Cyanosis or head nodding & Present & 68.3 & 80.4 & 17.8 & 97.6 \\
\hline Inability to cry or respiratory rate $\geqslant 90$ breaths $/ \mathrm{min}$ or head nodding & Present & 69.8 & 78.6 & 16.9 & 97.7 \\
\hline
\end{tabular}


hypoxaemia and supplemental oxygen therapy improves the outcome in severe pneumonia. ${ }^{5}$ Our study has shown that certain signs and symptoms in children with acute lower respiratory tract infections can be used to predict hypoxaemia. A rapid respiratory rate has been evaluated for predicting hypoxaemia or pneumonia in previous studies. ${ }^{6-10} 17$ A rapid respiratory rate, however, is likely to be affected by altitude and the presence of anaemia or fever, so its use as a predictor of hypoxaemia in a malaria endemic area needs to be considered with caution. ${ }^{20}$ Our study found that the presence of malaria parasitaemia, pallor, or fever had no effect on the relation between respiratory rate and hypoxaemia. Respiratory rate remained a useful predictor of hypoxaemia in a malaria endemic area, but the prevalence of parasitaemia in our predominantly urban population was low.

We found that several physical signs that could be taught easily to health workers and mothers were more closely associated with hypoxaemia than auscultatory signs. Cyanosis, a rapid respiratory rate, grunting, an inability to feed, impaired consciousness, head nodding, not crying during examination, and hepatomegaly $>2 \mathrm{~cm}$ were significantly associated with hypoxaemia. These signs used alone, however, were either not sufficiently sensitive or not specific. Cyanosis is a useful sign of hypoxaemia, but it is an infrequent, late, and subtle sign that can easily be missed in the presence of anaemia and in pigmented races. ${ }^{14}{ }^{18}$ Cyanosis is an ominous sign when present. ${ }^{19}$ In contrast, head nodding, which is caused by using the accessory muscles of respiration, is easily recognisable. This study, as well our previous study, found head nodding to be a useful sign. In the present study, it identified over half the children who were hypoxaemic, and its absence identified about nine out of 10 children who were not hypoxaemic. ${ }^{21}$ It may be argued that head nodding is useful as a predictor of hypoxaemia only in young children with acute lower respiratory tract infections, since it is an uncommon sign in children older than 5 years. Because head nodding is easily recognisable, however, it is a valuable sign in an age group that is most susceptible to hypoxaemia.

Since there are minimal adverse effects associated with oxygen therapy, it can be argued that in settings with adequate resources a very sensitive test is required. However, in many developing countries, oxygen therapy is expensive and available only in urban referral centres. It costs about US\$10 (£6) per

Key messages

- Simple physical signs that can be taught easily to healthcare workers can be used to identify hypoxaemic children who require referral or oxygen therapy, or both

- Hypoxaemia is associated with an increase in mortality in children with acute lower respiratory tract infections

- The presence of pneumonia on chest $x$ rays is not associated with hypoxaemia

- Pneumococcal infection is found more frequently among hypoxaemic than non-hypoxaemic children

- Malaria parasitaemia does not increase the rate of hypoxaemia in children with respiratory infections day to maintain one child on oxygen therapy at a flow rate of $1 \mathrm{l} / \mathrm{min}$. This is a cost many countries cannot afford. For this reason, careful decisions about oxygen therapy and referral are required to avoid overburdening the referral system and depleting scarce oxygen supplies. In settings such as the Gambia, with limited resources, guidelines requiring high specificity are desirable because of the potential costs. It is also valuable to have guidelines based on practical and easily recognisable signs.

Our study was performed on a cohort of children who were under close surveillance for H influenzae type $\mathrm{b}$ disease, and this may explain partially the low prevalence of hypoxaemia in the study population and the lower mortality rate than that observed in a Kenyan study. ${ }^{6}$ Moreover, we excluded infants $<2$ months of age, as they were too young to be included in the vaccine trial, and they may have been more prone to hypoxaemia. As in Kenya, a hypoxaemic child in the Gambia is five times more likely to die than a non-hypoxaemic child. Thus there is a need for the early recognition of signs of hypoxaemia and prompt treatment. Our model of cyanosis, a respiratory rate $\geqslant 70$ breaths/min, grunting, head nodding, an absence of a history of fever, and no spontaneous movement during examination, was the best predictor of hypoxaemia. The combination of head nodding and cyanosis identified seven out 10 hypoxaemic children, and its absence correctly identified four out of five non-hypoxaemic children, thereby minimising wastage of a precious resource. The addition of any other sign, as in our previous study, improved sensitivity but compromised specificity. The current recommendations for use of oxygen as suggested by the World Health Organisation would have led to two out of five children receiving oxygen when they did not have hypoxaemia. ${ }^{21}$ A combination of four signs for predicting hypoxaemia, as used in Papua New Guinea, performed as well as our model from the Gambia. ${ }^{21}$ Both Kenyan models, however, which had high sensitivities but low specificities, would have led to a high wastage of oxygen. ${ }^{6}$ All models had very low predictive values, which show the limitation of using clinical signs and also the low prevalence of hypoxaemia in the study population. The poor predictive value of clinical signs and poor agreement between observers are major limitations to the use of clinical signs for predicting hypoxaemia. ${ }^{25-27}$ Pulse oximetry is too costly for many hospitals in developing countries, therefore the selection of patients for referral or oxygen therapy has to be based on practical clinical signs that can be recognised readily by health workers with minimal expertise. Models that rely on the presence of cyanosis are not practical because of difficulties in assessing this sign. In circumstances where cyanosis may not be correctly assessed, the presence of head nodding, the inability to cry, and a respiratory rate $\geqslant 90$ breaths $/ \mathrm{min}$ are useful ways of predicting hypoxaemia.

Children with lower respiratory tract infections caused by invasive bacteria usually present with more severe disease than those with upper respiratory tract infections caused by viruses. Our data suggest that children with respiratory disease caused by invasive bacteria are more at risk of hypoxaemia than those with disease caused by respiratory syncytial virus. This is a surprising finding as clinical experience suggests 
that severe infection with respiratory syncytial virus is a common cause of hypoxaemia in infants.

Our study has shown that clinical signs requiring minimal basic skills and training to recognise can be used for formulating guidelines on the rational use of oxygen. These signs can also be used to identify those children with an acute lower respiratory tract infection requiring referral to a specialist centre.

We thank nursing staff from the Royal Victoria Hospital and Medical Research Council Laboratories for the care of the children, and Drs Ayo Palmer, Mariatou Jallow, Godwin Enwere, Abiodun Adegunloye, Tumani Corrah, and Anthony Juwah for contributing to the children's management

Contributors: MW initiated the research and helped design the study protocol. KM, the principal investigator of the Hib vaccine trial, helped design the study protocol. BG participated in the design of the study, and interpreted and reviewed the paper. $\mathrm{AO}, \mathrm{CO}$, and $\mathrm{SU}$ were involved with the design and execution of the study, data collection, data documentation, and clinical evaluation of the children. SJ and SU were involved with analysis and interpretation of data. RA performed the microbiological tests and also participated in the review of the paper. The paper was written jointly by SU, MW, KM, SJ, and BG. SU and MW will act as guarantors for the paper.

Funding: $\mathrm{SJ}$ is supported by an MRC grant. The vaccine trial was funded by the United States Agency for International Development Public Health Interagency for Vaccine Development and Health Research, the World Health Organisation programme for control of acute respiratory infections, the United Nations Children's Fund (Unicef), the Children's Initiative, and the United Nations development programme.

Conflict of interest: None.

1 Loweski J. Mortality from acute respiratory infections in children less than 5 years of age: global estimates. World Health Stat Q 1986;5:247-52.

2 Denny F, Loda FA. Acute respiratory infections are the leading cause of death in children in developing countries. Am J Trop Med Hyg $1986 ; 35: 1-2$.

3 Greenwood BM, Greenwood AM, Bradley AK, Tulloch S, Hayes R, Old field FSJ. Deaths in infancy and early childhood in a well-vaccinated, rural, West African population. Ann Trop Paediatr 1987;7:91-9.

4 Sazawal S, Black RE. Meta-analysis of intervention trials on casemanagement of pneumonia in community settings. Lancet 1992;340:52833.

5 World Health Organisation programme for the control of acute respiratory infections. Acute respiratory infections in children: case management in small hospitals in developing countries. Geneva: WHO/ARI $90.5,1990$.

6 Onyango FE, Steinhoff MC, Wafula EM, Wariua S, Musia J, Kitonyi J. Hypoxaemia in young Kenyan children with acute lower respiratory infection. BMJ 1993;306:612-4
7 Levene S, Mckenzie SA. Pulse oximetry in children. Lancet 1988;i:415-6.

8 Reuland DS, Steinhoff MC, Gilman RH, Bara M, Olivares EG, Jabra A, et al. Prevalence and prediction of hypoxemia in children with respiratory infections in the Peruvian Andes. J Pediatr 1991;119:900-7.

9 Lozano JM, Steinhoff M, Ruiz JG, Mesa ML, Martinez N, Dussan B. Clinical predictors of acute radiological pneumonia and hypoxaemia at high altitude. Arch Dis Child 1994;71:323-7.

10 Cherian T, John TJ, Simoes E, Steinhoff MC, John M. Evaluation of simple clinical signs for the diagnosis of acute lower respiratory tract infection. Lancet 1988;: 125-8.

11 Spooner V, Barker J, Tulloch S, Lehman D, Marshall T, Kajoi M, et al Clinical signs and risk factors associated with pneumonia in children admitted to Goroka hospital, Papua New Guinea. J Trop Pediat 1989;35:295-300.

12 Dyke T, Lewis D, Heegaard W, Manary M, Flew S, Rudeen K. Predicting hypoxia in children with acute lower respiratory infection: a study in the highlands of Papua New Guinea.J Trop Pediatr 1995;41:196-201.

13 Shann F, Barker J, Poore P. Clinical signs that predict death in children with severe pneumonia. Pediatr Infect Dis J 1989;8:852-5.

14 Stadie WC. The oxygen of the arterial and venous blood in pneumonia and its relation to cyanosis. J Exp Med 1919;30:215-40.

15 Mulholland EK, Olinsky A, Shann FA. Clinical findings and severity of acute bronchiolitis. Lancet 1990;335:1259-61.

16 Reynolds EOR. Arterial blood gas tensions in acute disease of lower respiratory tract in infancy. $B M J$ 1963;1:1192-5.

17 Hall CB, Hall WJ, Speers DM. Clinical and physiological manifestations of bronchiolitis and pneumonia. Outcome of respiratory syncytial virus. Am J Dis Child 1979;133:798-802.

18 Simpson H, Flenley DC. Arterial blood-gas tensions and $\mathrm{pH}$ in acute lower-respiratory-tract infections in infancy and childhood. Lance $1967 ; 1: 7-12$.

19 Dyke T, Brown N. Hypoxia in childhood pneumonia: better detection and more oxygen needed in developing countries. BMJ 1994;308:119-20.

20 O'Dempsey TJD, Todd J. Respiratory rates are poor predictors of hypoxaemia. BMJ 1993;306:1342.

21 Weber MW, Usen S, Palmer A, Jaffar S, Mulholland EK. Predictors of hypoxaemia in hospital admissions with acute lower respiratory tract infection in a developing country. Arch Dis Child 1997;76:1-5.

22 Mulholland EK, Hilton S, Adegbola RA, Usen S, Oparaugo A, Omosigho $\mathrm{C}$, et al. Randomised trial of Haemophilus influenzae type b-tetanus protein conjugate for prevention of pneumonia and meningitis in Gambian infants. Lancet 1997;349:1191-7.

23 Cane PA, Pringle CR. Evolution of subgroup A respiratory syncytial virus: evidence for progressive accumulation of amino acid changes in the attachment protein J Virol 1995:69:2918-25.

24 Seaton A, Seaton D, Leitch AG. Functions of the respiratory tract. In Crofton and Douglas's Respiratory diseases, 4th edn. Oxford: Blackwell Scientific, 1989.

25 Margolis PA, Ferkol TW, Marsocci S, Super DM, Keyes LL, McNutt R, et al. Accuracy of the clinical examination in detecting hypoxemia in infants with respiratory illness. J Pediatr 1994;124:552-60.

26 Spiteri MA, Cook DG, Clarke SW. Reliability of eliciting physical signs in examination of the chest. Lancet 1988;1:873-5.

27 Wang EE, Milner RA, Navas L, Maj H. Observer agreement for respiratory signs and oximetry in infants hospitalised with lower respiratory infections. Am Rev Respir Dis 1992;145:106-109.

(Accepted 28 October 1998)

\author{
Abstract \\ Objective To assess the relation between male and \\ female medical leadership. \\ Design Cross sectional study on predictive factors for \\ female medical leadership with data on sex, age, \\ specialty, and occupational status of Norwegian \\ physicians. \\ Setting Oslo, Norway. \\ Subjects 13844 non-retired Norwegian physicians. \\ Main outcome measure Medical leaders, defined as \\ physicians holding a leading position in hospital \\ medicine, public health, academic medicine, or private \\ health care.
}

Results $14.6 \%$ (95\% confidence interval $14.0 \%$ to $15.4 \%$ ) of the men were leaders compared with $5.1 \%$ $(4.4 \%$ to $5.9 \%)$ of the women. Adjusted for age men had a higher estimated probability of leadership in all categories of age and job, the highest being in academic medicine with 0.57 (0.42 to 0.72$)$ for men aged over 54 years compared with 0.39 (0.21 to 0.63 ) for women in the same category. Among female hospital physicians there was a positive relation between the proportion of women in their specialty and the probability of leadership.

Conclusion Women do not reach senior positions as easily as men. Medical specialties with high proportions of women have more female leaders.
Editorial

by Showalter

Department of Otorhinolaryngology,

Ullevål University Hospital, N-0407 Oslo, Norway Kari J Kværner, associate professor continued over

BMJ 1999;318:91-4 